\title{
Molecular Characterization of Olfactory Bulb Neural Stem Cells during Proliferation and Differentiation
}

\author{
Entsar A. Saad", Hany E. S. Marei², Mohammed A. El-Magd³, Heba M. El-Fatiry' \\ 'Chemistry Department, Faculty of Science, Damietta University, Damietta, Egypt. \\ ${ }^{2}$ Department of Cytology and Histology, Faculty of Veterinary Medicine, Mansoura University, Mansoura, Egypt. \\ 'Department of Anatomy and Embryology, Faculty of Veterinary Medicine, Kafrelsheikh University, Egypt.
}

\begin{tabular}{l}
\hline ARTICLE INFO \\
\hline Article history: \\
Received on: 17/08/2017 \\
Accepted on: $22 / 11 / 2017$ \\
Available online: $28 / 01 / 2018$ \\
\hline Key words: \\
Neurons, Oligodendrocytes, \\
Astrocytes, Gene expression, \\
PCR
\end{tabular}

PCR

\begin{abstract}
Objective: This study aimed to demonstrate the fate of human olfactory bulb neural stem cells (hOBNSCs). Reportedly, these cells can be expanded in vitro under prolonged mitogen stimulation without propensity to transform. Material and methods: We assessed their possible ability to proliferate and differentiate into different neurons, oligodendrocytes and astrocytes through monitoring changes in expression profile of proliferation (NES, NR4A1, SOX2, MSI1) and differentiation (FOXO4, CSPG4, MAP2, GFAP)-related genes. Results: In vitro induction of hOBNSCs proliferation by addition of basic fibroblast growth factor (bFGF), epidermal growth factor (EGF), and leukemia inhibitory factor (LIF) to basal serum-free medium (DMEM/F12) resulted in significant up-regulation of proliferation-related genes. Differentiation of hOBNSCs, which was initiated by replacing bFGF and EGF by triiodothyronine (T3), significantly increased expression of differentiation-related genes. Among the differentiated cells, GFAP-expressing astrocytes constituted the highest population of cells followed by CSPGS-expressing immature oligodendrocytes, then MAP2-expressing immature neurons, and finally FOXO4-expressing mature oligodendrocytes. Conclusion: These data will enable us to understand the mechanism of proliferation and differentiation of hOBNSCs before and after their engraftment during cell-based therapy for neurodegenerative diseases.
\end{abstract}

\section{INTRODUCTION}

Stem cells (SCs) are defined as cells having the ability to self-renew, and to differentiate into multiple phenotypic lineages (Tuszynski et al., 1996). Recently, there has been an increasing interest for their use in various biochemical applications. However SCs heterogeneity causes a significant obstacle to the clinical implementation of cell-based therapies (Young et al., 2016). In humans, neural SCs (NSCs) have successfully been isolated from the olfactory bulb (OB) (Marei et al., 2012). Thereby, human adult olfactory bulb NSCs (hOBNSCs) provide an attractive tool for transplantation-based therapy of neurodegenerative diseases that avoids the ethical and moral questions associated with the use of human embryonic or heterologous material (Casalbore et al., 2009; Marei et al., 2016).

${ }^{*}$ Corresponding Author

Entsar A. Saad, Chemistry Department, Faculty of Science, Damietta University, Damietta, Egypt.

E-mail: entsarsaad@gmail.com
In vitro, NSCs can be induced to proliferate when they are exposed to growth factors (Vescovi et al., 1993). In the OB, NSCs differentiate into inter-neurons in the granular and glomerular layer. Although none of the markers are completely exclusive for NSC, some of them including nestin, NR4A1, SOX2, and MSI1, are highly enriched in SC populations (Nakano et al., 2005). Nestin is a type VI intermediate filament protein responsible for mechanical integrity of the cell and involved in formation of tissue architecture and in tissue regeneration (Neradil and Veselska, 2015). Upon differentiation, nestin is down-regulated and substituted by other intermediate filament proteins such as glial fibrillary acidic protein (GFAP) in astrocytes or neurofilaments in neuronal cells (Michalczyk and Ziman, 2005). The orphan nuclear hormone receptor NR4A1 is involved in cell survival and apoptosis ( $\mathrm{Li}$ et $a l ., 2006)$. SOX2 is a transcription factor essential for maintaining self-renewal of undifferentiated embryonic SCs (Pevny and Nicolis, 2010). However, SOX2 is down-regulated during differentiation when they become post mitotic (Suh et al., 2007). The MSI1 
RNA-binding protein is a specific marker for stem/progenitor cells of neural origin (Uren et al., 2015). Neural progenitors express high levels of MSI1, which is rapidly turned off during terminal differentiation, and its expression is virtually absent in post-mitotic neurons (Glazer et al., 2012).

Mature neurons have distinctive and definite morphological criteria. However, it is usually hard to undoubtedly recognize a cell as being a neuron in vitro or in vivo (Nakano et al., 2005). Microtubule-associated protein 2 (MAP2) is involved in maintenance of neuronal morphology, development of dendrite, and outgrowth of oligodendrocyte and neurite (Lim and Halpain, 2000; Zamora-Leon et al., 2001). Similar to neurons, mature oligodendrocytes have a distinctive morphology. However, in vitro, oligodendrocytes may not be easily distinguishable from other process-bearing cells. Markers used to identify oligodendrocytes for instance are the forkhead box protein $\mathrm{O} 4$ (FOXO4) and chondroitin sulfate proteoglycan 4 (CSPG4) (Nakano et al., 2005). FOX proteins contribute in cellular proliferation, transforma- tion, differentiation and longevity (Tsai et al., 2007). CSPG4 cells have the ability to generate oligodendrocytes in vitro as well as in vivo, and so they are often called oligodendrocyte precursor cells (Ozerdem et al., 2001; Schiffer et al., 2017). In vivo, astrocytes appear stellate and in vitro they appear flattened polygonal or have long processes. Glial fibrillary acidic protein (GFAP), an intermediate filament protein present approximately exclusively in astrocytes, is used to identify astrocytes in vivo and in vitro (Nakano et al., 2005). Together with actin filaments and microtubules, they form an integrated system that regulates many cellular processes, such as cell morphology and proliferation (Moeton et al., 2016).

This study aimed to demonstrate the fate of hOBNSCs through assessment of their ability to proliferate and differentiate into different neurons, oligodendrocytes and astrocytes and to detect gene expression profile that is specific for each cell lineage during proliferation and differentiation processes.

Table 1: Human adult olfactory bulb neural stem cells (hOBNSCs).

\begin{tabular}{|c|c|c|c|}
\hline Sample index & Age/Sex & Diagnosis & Source \\
\hline OB1 & $45 / \mathrm{M}$ & Olfactory groove meningioma & Institute of Neurosurgery, Catholic University School of Medicine, Rome, Italy \\
\hline OB2 & $39 / \mathrm{M}$ & Anterior communicating artery aneurysm & Institute of Neurosurgery, Catholic University School of Medicine, Rome, Italy \\
\hline
\end{tabular}

\section{MATERIAL AND METHODS}

\section{Isolation and culturing of hOBNSCs}

The olfactory bulbs (OBs) were collected from two patients undergoing craniotomy at the Institute of Neurosurgery, Catholic University, Rome, Italy (Table 1). Prior signed agreements were obtained according to protocols approved by the Ethical Committee of the Catholic University. The experiments were undertaken with the understanding and written consent of each subject, and the study conforms with World Medical Association Declaration of Helsinki. Obtained OBs were directly broken down at $37{ }^{\circ} \mathrm{C}$ in $0.1 \%$ Papain (Sigma-Aldrich, St. Louis, MO, USA) for $30 \mathrm{~min}$. Resultant cells were then grown in basal serum-free medium (DMEM/F12; Invitrogen, Carlsband, CA, USA) that contain progesterone $0.025 \mathrm{mg} / \mathrm{mL}$, L-glutamine $2 \mathrm{mM}$, glucose $0.6 \%$, sodium selenite $5.2 \mathrm{ng} / \mathrm{mL}$, insulin $0.025 \mathrm{mg} / \mathrm{mL}$, apo-transferrin sodium salt $0.1 \mathrm{mg} / \mathrm{mL}$, putrescine $9.6 \mu \mathrm{g} / \mathrm{mL}$, sodium bicarbonate 3 $\mathrm{mM}$, Hepes $5 \mathrm{mM}$, BSA $4 \mathrm{mg} / \mathrm{mL}$ and heparin $4 \mu \mathrm{g} / \mathrm{mL}$ in the presence of $20 \mathrm{ng} / \mathrm{mL}$ epidermal growth factor (EGF; PeproTech, Rocky Hill, NJ, USA), 10 ng/mL basic fibroblast growth factor (bFGF; PeproTech, Rocky Hill, NJ, USA), and $20 \mathrm{ng} /$ $\mathrm{mL}$ leukemia inhibitory factor (LIF; Immunological Sciences, Rome, Italy). For $4 \mathrm{~min}$ at $37^{\circ} \mathrm{C}$ the primary neurospheres were broken down using accutase (Invitrogen) then diluted and plated (single cell/mini-well) onto 96 -well plates. Existence of one single cell in mini-wells was confirmed via microscopic examination. After 7 days these wells were examined for secondary neurosphere generation. Secondary neurospheres were dissociated then cultured $\left(10^{3} \mathrm{cells} / \mathrm{cm}^{2}\right)$ in serum-free medium containing EGF and bFGF, and passaged up to P30. Parallel cultures of cells grown as adherent mono-layers were confirmed between P7 and P10 in medium containing EGF and bFGF with 5\% fetal calf serum supplement (Hyclone, Logan, UT). Differentiation investigations were done next to 5 days after culturing $10^{5}$ proliferated cells which were plated in 24-well plate supplied with a poly-L-lysine coated glass cover slips in presence of $1 \%$ fetal calf serum (Hyclone) supplemented with cAMP (50 mM), all-trans retinoic acid (5 mM) and T3 (30 $\mathrm{nM}$ ) (Sigma-Aldrich, St. Louis, MO, USA) and in absence of EGF and $b F G F$.

\section{Morphological examination}

hOBNSCs cells were plated on 12 -well plates at $2 \times$ $104 /$ well, and allowed to reach $70 \%-80 \%$ confluence. All cultures were incubated at $37{ }^{\circ} \mathrm{C}$ in an atmosphere containing $5 \%$ $\mathrm{CO}_{2}$ for $24 \mathrm{~h}$. After this, cells were washed 3 times with $1 \mathrm{X}$ PBS (ice cold), fixed for 30 min with $4 \%$ formaldehyde solution in PBS and the morphologic changes of hOBNSCs cells were examined and photographs were taken using an inverted microscope (Nikon, Japan) at 40X magnification. For confirmation the experiment was repeated three times with nearly similar results.

\section{Detection of cell viability by MTT assay}

Cell viability of differentiated hOBNSCs was assessed using conventional MTT assay. The cultured cells in 96-well plates $\left(5 \times 10^{3} /\right.$ well $)$ were incubated with $20 \mu \mathrm{L}$ of MTT stock solution $(5 \mathrm{mg} / \mathrm{mL})$ for $4 \mathrm{~h}$ at $37{ }^{\circ} \mathrm{C}$. Absorbance of extracted resultant MTT formazan with $150 \mu \mathrm{L}$ dimethyl sulfoxide (DMSO) was recorded at $570 \mathrm{~nm}$ using a microplate reader (Bio-Teck, USA). The absorbance of untreated cells was set to $100 \%$, and the results for each treated sample are expressed as the percentage of MTT reduction. 


\section{Detection of relative gene expression by real-time PCR}

Isolation of hOBNSCs' total RNA was performed using Gene JET RNA Purification Kit (Thermo Scientific, \# K0731, USA) and RevertAid H Minus Reverse Transcriptase (Thermo Scientific, \#EP0451, USA) was used to reverse transcribe isolated total RNA ( $5 \mu \mathrm{g} / \mathrm{sample}$ ) into cDNA. cDNA was then used as a template to determine the relative expression of the candidate genes using StepOnePlus real time PCR system (Applied Biosystem, USA). Primer 5.0 software was used for primers (Table 2) designation. The reference used to calculate the fold change in target gene expression was the housekeeping gene GAPDH. cDNA template $(2 \mu \mathrm{L})$ was mixed with $2 \mathrm{X}$ Maxima SYBR Green/ROX qPCR Master Mix (Thermo Scientific, \# K0221, USA) $(12.5 \mu \mathrm{L})$, forward primer $(1 \mu \mathrm{L})$, reverse primer $(1 \mu \mathrm{L})$, and nuclease free water $(8.5 \mu \mathrm{L})$ to form $25 \mu \mathrm{L}$ of PCR mix. Thermal cycling conditions included initial denaturation at $95^{\circ} \mathrm{C}$ for $10 \mathrm{~min}, 40-45$ cycles of amplification of DNA denaturation at $95{ }^{\circ} \mathrm{C}$ for $15 \mathrm{~s}$, annealing at $60{ }^{\circ} \mathrm{C}$ for $30 \mathrm{~s}$ and extension at $72{ }^{\circ} \mathrm{C}$ for $30 \mathrm{~s}$. The temperature was increased at the end of the last cycle from 63 to $95{ }^{\circ} \mathrm{C}$ for analysis of the melting curve. Target genes and GAPDH gene cycle threshold $(\mathrm{Ct})$ values were calculated then $2^{-\Delta \Delta \mathrm{Ct}}$ method was used to determine relative gene expression.

\section{Statistical analysis}

Results were expressed as means \pm standard error of the mean (SEM). One-way analysis of variance (ANOVA) was used to evaluate the statistical significance using SPSS software version 18.0. Duncan's multiple range test (DMRT) was used for the individual comparisons. $\mathrm{P}<0.05$ was considered statistically significant.

Table 2: Sequences of forward and reverse primers used in real-time PCR.

\begin{tabular}{|c|c|c|}
\hline Gene & $\begin{array}{c}\text { Forward primer } \\
((5-3)\end{array}$ & $\begin{array}{c}\text { Reverse primer } \\
(5-3)\end{array}$ \\
\hline NES & GGGCCTACAGAGCCAGATCG & CAGGAGGGTCCTGTACGTGG \\
\hline NR4A1 & CCATCCAGAGTGAGATGCCC & TGGGCTTGATGAACTCAGGG \\
\hline SOX2 & GGATAAGTACACGCTGCCCG & CTGTCCATGCGCTGGTTCAC \\
\hline MSI1 & ACGACCCCTGCAAGATGTTC & CCCGAACTGGCCGAAGTATT \\
\hline FOXO4 & CTGGGGGAAAAGGCCATTGA & GTGGCGGATCGAGTTCTTCC \\
\hline CSPG4 & CACAGAGGAACCCTGGATGG & CTTCAGCGAGAGGAGCACTT \\
\hline MAP2 & GGGTGCATCCAGTTTCTGCG & CCCAATCAATGCTTCCTCGGT \\
\hline GFAP & GCTCAATGTCAAGCTGGCCC & CTTTTGCCCCCTCGAATCTGC \\
\hline GAPDH & GTT CGA CAG TCA GCC GCA TC & CGA CCA AAT CCG TTG ACT CCG \\
\hline
\end{tabular}

\section{RESULTS}

\section{Morphological characters of the proliferated and differenti- ated hOBNSCs}

The proliferated hOBNSCs formed clusters of cells called neurospheres which contained undifferentiated NSCs, as revealed by the lack of any specific morphology (Figure1A and B). The formed neurospheres were in form of either free floating or loosely attached growing spheres. These cells generated clonally related progeny that differentiated into neurons, oligodendrocytes, and astrocytes. The in vitro culture showed that a common progenitor existed for oligodendrocytes, astrocytes, and unipolar single neuron cell in the second differentiation day (Figure 1D) which converted into multipolar neuron by forming synaps with other neurons (Figure 1E).

\section{Effects of differentiation media on cell viability of hOBNSCs}

MTT assay was used to evaluate the effect of the different differentiation compounds (3'-5'-cyclic adenosine monophosphate (cAMP) $50 \mathrm{mM}$, all-trans retinoic acid $5 \mathrm{mM}$, triiodothyronine (T3) $30 \mathrm{nM}$, and ascorbic acid) on cell viability of hOBNSCs. Obtained results showed that the percent of cell viability following addition of proliferation media $(90 \pm 10)$ did not significantly differ from that of differentiation media $(80 \pm$ 7.0) (Figure 2).

\section{Relative expression of proliferation-related genes in hOBN-} SCs

Relative expression of the 4 proliferation-related genes (NES, NR4A1, SOX2, and MSI1) was determined in hOBNSCs by real-time PCR (Figure 3). A significant $(\mathrm{P}<0.05)$ up-regulation in expression of these genes was detected in the proliferated hOBNSCs as compared to control group (MSI1 gene in differentiated cells). SOX2 gene showed the highest significant up-regulation level as compared to other proliferative genes. However, MSI1 gene showed the lowest expression level. No significant difference was detected in the expression of NES and NR4A1 genes $(\mathrm{P}>0.05)$.

\section{Relative expression of differentiation-related genes in hOBN- SCs}

To check whether hOBNSCs had the capability to differentiate into both neuronal and glial cell types, primary neurospheres were dissociated into single cells and induced to differentiate in medium lacking mitogens. Data obtained from qPCR revealed a significant $(\mathrm{P}<0.05)$ up-regulation in expression level of the 4 differentiation-related genes (FOXO4, CSPGS, MAP2, and GFAP) in hOBNSCs as compared to control group (FOXO4 gene in proliferative cells) (Figure 4). GFAP gene showed the highest significant up-regulation level as compared to other differentiated genes. However, FOXO4 gene showed the lowest expression level. No significant difference was observed between 
the expression of MAP2 and CSPGS genes $(\mathrm{P}>0.05)$. Thus, it is differentiated to neurons, astrocytes, and oligodendrocytes. likely that the $\mathrm{OB}$ harbors multipotent progenitor cells that can be


Fig. 1: Morphological characters of proliferated and differentiated hOBNSCs. (A) Neurospheres formed after one week of proliferation. (B) Typical neurospheres indicating the high proliferation ability of cultured cells. (C) First hours in the first day of differentiation showing perfect single cells. (D) Second day differentiation showing unipolar neuron differentiated from single cells. (E) The third day differentiation showing multipolar neuron making synapse to another neuron.

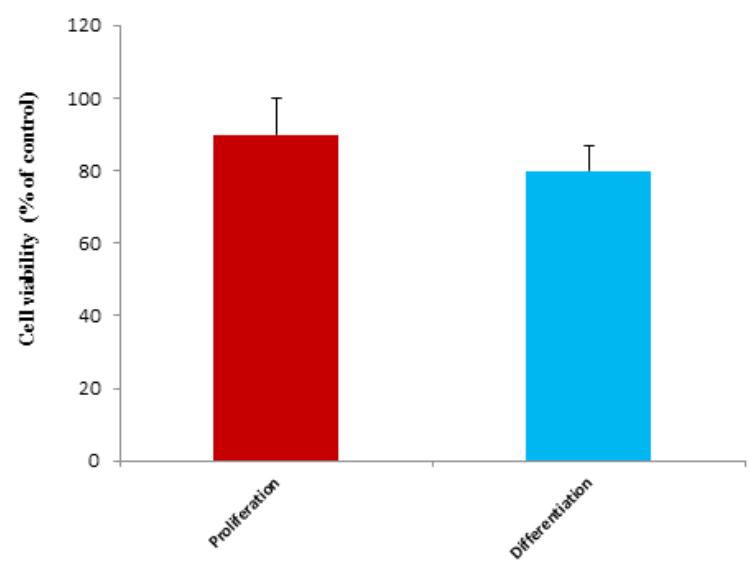

Fig. 2: Effects of differentiation media on human olfactory bulb neural stem cells (hOBNSCs) viability using MTT assay. Data are represented as mean \pm SEM $(\mathrm{n}=8$ ).

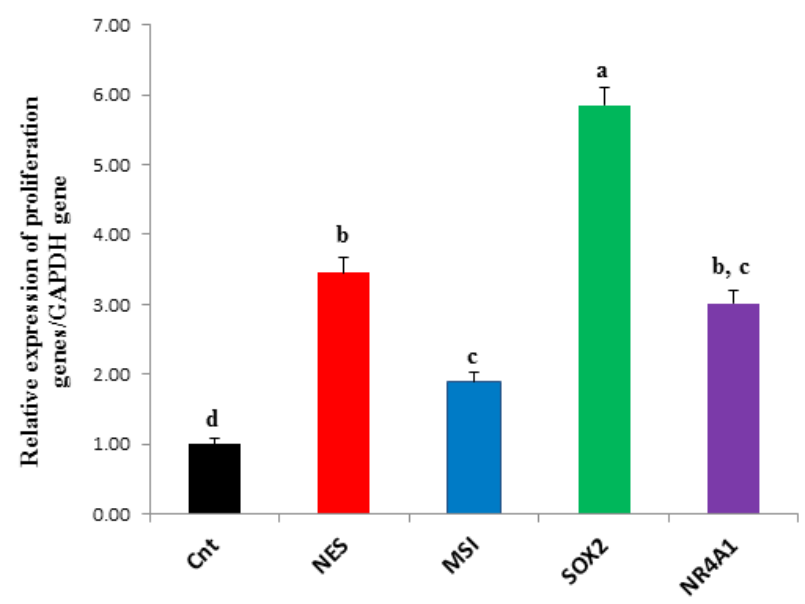

Fig. 3: Real-time quantitative PCR analysis of the expression of proliferation related genes in human olfactory bulb neural stem cells (hOBNSCs) as compared to control (Cnt). Values are expressed as means \pm SEM (each group was tested in triplicate). Means carrying different superscript letters are significantly different $(\mathrm{P}<0.05)$. 


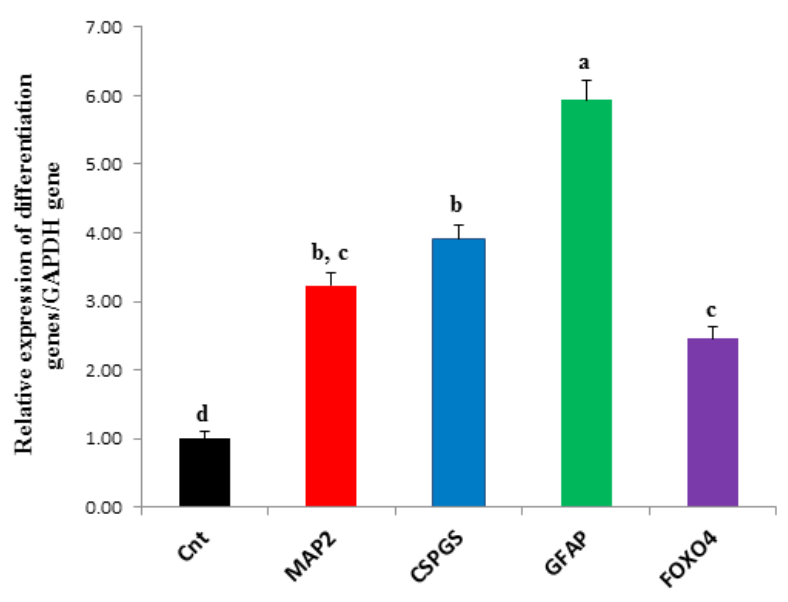

Fig. 4: Real-time quantitative PCR analysis of the expression of differentiation related genes in human olfactory bulb neural stem cells (hOBNSCs) as compared to the control (Cnt). Values are expressed as means \pm SEM (each group was tested in triplicate). Means carrying different superscript letters are significantly different $(\mathrm{P}<0.05)$.

\section{DISCUSSION}

The main aim of the present study was to provide more details about the changes in the expression profile of some proliferation- and differentiation-related genes in hOBNSCs following addition and removal, respectively, of some trophic agents. Isolation of NSCs from the hOB has some advantages if compared to other sources of stem cells as induced pluripotent stem cells (iPSCs), and human embryonic stem cells (hESCs). Human OBNSC provides less active mature adult cells which might exclude the rapid proliferation and potential tumorigenic potential of hESCs and iPSCs (Isobe et al., 2014; Suzuki et al., 2014). Besides, hESCs-derived oligodendrocytes precursor cells need more cytokines, demanding techniques, and longer time for differentiation (Hu et al., 2009; Sundberg et al., 2010). Thus, in vitro differentiation of adult hOBNSCs could be a promising tool to overcome some of the practical and ethical concerns of fetal tissue transplantation in neurodegenerative diseases.

Stopping the addition of neurotrophic agents, EGF, bFGF, and LIF inhibits hOBNSCs proliferation, but favors for differentiation. To check whether this withdrawal can affect cell viability, MTT assay was conducted and its results showed no significant effect on cell viability. This means that neither neurotrophic agents' withdrawal nor addition to differentiation-induced factors has any effect on hOBNSCs cell viability but rather they directed the cells toward differentiation.

During proliferation phase, hOBNSCs have an increased level in the expression of proliferation-related genes (NES, NR4A1, SOX2, and MSI1) compared to control group (MSI1 gene in differentiation cells). Among these 4 genes, SOX2 showed the highest significant up-regulation level. In consistent with our findings, a previous study reported early activation of SOX2 in NSCs derived from adult human OB (Alizadeh et al., 2017) and in the embryonic nervous system, predominantly in the proliferating, undifferentiated precursors (Pevny and Nicolis, 2010). Unlike SOX2, MSI1 gene, which is a marker for neuronal progenitor cells (Uren et al., 2015), showed the lowest expression level. Thus, we would expect a decrease in the proliferation of neuronal progeni- tors as compared to other progenitors. The other two markers for undifferentiated neural progenitors, NES and NR4A1 showed a moderate up-regulation in the proliferated OBNSCs.

During differentiation phase, there is an up-regulation in expression level of the all 4 differentiation-related genes (FOXO4, CSPGS, MAP2, and GFAP) in hOBNSCs as compared to the control (FOXO4 gene in the proliferative cells). The astrocytes marker GFAP showed the highest significant up-regulation level as compared to other differentiation genes. In agreement with our results, it has been documented that during differentiation Nestin is down-regulated and replaced by GFAP in astrocytes (Michalczyk and Ziman, 2005). Therefore, the astrocytes may constitute the highest percent of the differentiated OBNSCs. The expression of GFAP and its polymerization to gliofilaments are important hallmarks of the process of astroglial morphological differentiation (Messam et al., 2000). On the other hand, FOXO4 gene, which is a marker for mature oligodendrocytes, showed the lowest expression level. This suggests the presence of few numbers of mature oligodendrocytes than astrocytes in the differentiated hOBNSCs. Moderate expression was observed for MAP2 (which is a marker for immature neurons) followed by CSPGS (which is a marker for immature oligodendrocytes). In vitro CSPG4 cells represent multipotent cells, since they are capable of generating oligodendrocytes, neurons and astrocytes in the presence of specific growth factors. However, in vivo, CSPG4 cells in the adult intact central nervous system produce only CSPG4 cells and oligodendrocytes (Honsa et al., 2016).

\section{CONCLUSION}

Data obtained from the differential expression of differentiation- related genes revealed that hOBNSCs can be differentiated into a large variety of neural cells. Among these cells, GFAP-expressing astrocytes constituted the highest population of cells followed by CSPGS-expressing immature oligodendrocytes, then MAP2-expressing immature neurons, and finally FOXO4-expressing mature oligodendrocytes which constituted the lowest population of cells. These data will help us to control hOBNSCs 
proliferation and differentiation in vitro via addition/deletion of specific growth factors. Further investigations are also required to study the functional characterization of these in vitro formed mature cells.

\section{Financial support and sponsorship: Nil.}

Conflict of Interests: There are no conflicts of interest.

\section{REFERENCES}

Alizadeh R, Hassanzadeh G, Joghataei MT, Soleimani M, Moradi F, Mohammadpour S, Ghorbani J, Safavi A, Sarbishegi M, Pirhajati Mahabadi V, Alizadeh L, Hadjighassem M. In vitro differentiation of neural stem cells derived from human olfactory bulb into dopaminergic-like neurons. Eur J Neurosci, 2017; 45: 773-784.

Casalbore P, Budoni M, Ricci-Vitiani L, Cenciarelli C, Petrucci G. Tumorigenic potential of Olfactory Bulb-Derived Human Adult Neural Stem Cells Associates with Activation of TERT and NOTCH1. PLoS One, 2009; 4 (2): e4434.

Glazer RI, Vo DT, Penalva LO. Musashil: an RBP with versatile functions in normal and cancer stem cells. Front Biosci (Landmark Ed), 2012; 17: 54-64.

Honsa P, Valny M, Kriska J, Matuskova H, Harantova L, Kirdajova D, Valihrach L, Androvic P, Kubista M, Anderova M. Generation of reactive astrocytes from NG2 cells is regulated by sonic hedgehog. Glia, 2016; 64: 1518-1531.

Hu BY, Du ZW, Li XJ, Ayala M, Zhang SC. Human oligodendrocytes from embryonic stem cells: conserved SHH signaling networks and divergent FGF effects. Development, 2009; 136: 1443-1452.

Isobe K, Cheng Z, Nishio N, Suganya T, Tanaka Y, Ito S. iPSCs, aging and age-related diseases. N Biotechnol, 2014; 31: 411-421.

Li QX, Ke N, Sundaram R, Wong-Staal F. NR4A1, 2, 3--an orphan nuclear hormone receptor family involved in cell apoptosis and carcinogenesis. Histol Histopathol, 2006; 21: 533-540.

Lim RW, Halpain S. Regulated association of microtubule-associated protein 2 (MAP2) with Src and Grb2: evidence for MAP2 as a scaffolding protein. J Biol Chem, 2000; 275: 20578-20587.

Marei HE, Althani A, Rezk S, Farag A, Lashen S, Afifi N, Abd-Elmaksoud A, Pallini R, Casalbore P, Cenciarelli C, Caceci T. Therapeutic potential of human olfactory bulb neural stem cells for spinal cord injury in rats. Spinal Cord, 2016; 54: 785-797.

Marei HES, Ahmed AE, Michetti F, Pescatori M, Pallini R, Casalbore P, Cenciarelli C, Elhadidy M. Gene expression prolife of adult human olfactory bulb and embryonic neural stem cell suggests distinct signaling pathways and epigenetic control. PLoS One, 2012; 7 (4): e33542.

Messam CA, Hou J, Major EO. Coexpression of nestin in neural and glial cells in the developing human CNS defined by a human-specific anti-nestin antibody. Exp Neurol, 2000; 161: 585-596.

Michalczyk K, Ziman M. Nestin structure and predicted function in cellular cytoskeletal organisation. Histol Histopathol, 2005; 20 : 665-671.

Moeton M, Stassen OM, Sluijs JA, van der Meer VW, Kluivers LJ, van Hoorn H, Schmidt T, Reits EA, van Strien ME, Hol EM. GFAP isoforms control intermediate filament network dynamics, cell morphology, and focal adhesions. Cell Mol Life Sci, 2016; 73: 4101-4120.

Nakano I, Paucar AA, Bajpai R, Dougherty JD, Zewail A, Kelly TK, Kim KJ, Ou J, Groszer M, Imura T, Freije WA, Nelson SF, Sofroniew
MV, Wu H, Liu X, Terskikh AV, Geschwind DH, Kornblum HI. Maternal embryonic leucine zipper kinase (MELK) regulates multipotent neural progenitor proliferation. J Cell Biol, 2005; 170: 413-427.

Neradil J, Veselska R. Nestin as a marker of cancer stem cells. Cancer Sci, 2015; 106: 803-811.

Ozerdem U, Grako KA, Dahlin-Huppe K, Monosov E, Stallcup WB. NG2 proteoglycan is expressed exclusively by mural cells during vascular morphogenesis. Dev Dyn, 2001; 222: 218-227.

Pevny LH, Nicolis SK. Sox2 roles in neural stem cells. Int J Biochem Cell Biol, 2010; 42: 421-424.

Schiffer D, Annovazzi L, Bovio E, Mellai M. NG2 Proteoglycan in the Diagnosis, Prognosis and Therapy of Gliomas. Int J cell Sci \& mol boil, 2017; 2(2): 555582. DOI: 10.19080/IJCSMB.2017.02.555582.

Suh H, Consiglio A, Ray J, Sawai T, D’Amour KA, Gage FH. In vivo fate analysis reveals the multipotent and self-renewal capacities of Sox $2+$ neural stem cells in the adult hippocampus. Cell Stem Cell, 2007; 1: $515-528$.

Sundberg M, Skottman H, Suuronen R, Narkilahti S. Production and isolation of $\mathrm{NG} 2+$ oligodendrocyte precursors from human embryonic stem cells in defined serum-free medium. Stem Cell Res, 2010; 5: 91-103.

Suzuki DE, Nakahata AM, Okamoto OK. Knockdown of E2F2 inhibits tumorigenicity, but preserves stemness of human embryonic stem cells. Stem Cells Dev, 2014; 23: 1266-1274.

Tsai KL, Sun YJ, Huang CY, Yang JY, Hung MC, Hsiao CD Crystal structure of the human FOXO3a-DBD/DNA complex suggests the effects of post-translational modification. Nucleic Acids Res, 2007; 35: 6984-6994.

Tuszynski MH, Roberts J, Senut MC, HS U, Gage FH. Gene therapy in the adult primate brain: intraparenchymal grafts of cells genetically modified to produce nerve growth factor prevent cholinergic neuronal degeneration. Gene Therapy, 1996; 3(4): 305-314.

Uren PJ, Vo DT, de Araujo PR, Potschke R, Burns SC, Bahrami-Samani E, Qiao M, de Sousa Abreu R, Nakaya HI, Correa BR, Kuhnol C, Ule J, Martindale JL, Abdelmohsen K, Gorospe M, Smith AD, Penalva LO. RNA-Binding Protein Musashi1 Is a Central Regulator of Adhesion Pathways in Glioblastoma. Mol Cell Biol, 2015; 35: 2965-2978.

Vescovi AL, Reynolds BA, Fraser DD, Weiss S. bFGF regulates the proliferative fate of unipotent (neuronal) and bipotent (neuronal/astroglial) EGF-generated CNS progenitor cells. Neuron, 1993; 11: 951-966.

Young FI, Telezhkin V, Youde SJ, Langley MS, Stack M, Kemp PJ, Waddington RJ, Sloan AJ, Song B. Clonal Heterogeneity in the Neuronal and Glial Differentiation of Dental Pulp Stem/Progenitor Cells. Stem Cells Int, 2016; 2016: 1-10.

Zamora-Leon SP, Lee G, Davies P, Shafit-Zagardo B. Binding of Fyn to MAP-2c through an SH3 binding domain. Regulation of the interaction by ERK2. J Biol Chem, 2001; 276: 39950-39958.

How to cite this article:

Saad EA, Marei HES, El-Magd MA, El-Fatiry HM. Molecular Characterization of Olfactory Bulb Neural Stem Cells during Proliferation and Differentiation. J App Pharm Sci, 2018; 8 (01): 087-092. 